\title{
Research Square \\ Generation of Hollow Airy Beam by Superposing of Two-Dimensional Airy Beams
}

Marcelino Anguiano-Morales ( $\nabla$ manguiano@itchihuahua.edu.mx )

Instituto Tecnologico de Chihuahua https://orcid.org/0000-0002-1625-5492

\section{Research Article}

Keywords: Airy beams, Bessel beam, Non-diffracting beam

Posted Date: February 21st, 2022

DOI: https://doi.org/10.21203/rs.3.rs-1350425/v1

License: (c) (i) This work is licensed under a Creative Commons Attribution 4.0 International License. Read Full License 


\title{
Generation of hollow Airy beam by superposing of two-dimensional Airy beams
}

\author{
Marcelino Anguiano-Morales \\ División de Estudios de Posgrado e Investigación, Instituto Tecnológico de Chihuahua, Av. \\ Tecnológico No 2909, 31310 Chihuahua, Chih., México
}

\begin{abstract}
We study the collisions of four self-focused counterpropagating 2D Airy beams propagating in free space. Their interactions demonstrate that can be obtained hollow beams through the superposition of more than two Airy beams and are well-preserved upon propagation.
\end{abstract}

Keywords Airy beams, Bessel beam, Non-diffracting beam

* Corresponding author.

E-mail address: marcelino.am@ chihuahua.tecnm.mx (M. Anguiano-Morales).

\section{Introduction}

Of the invariant optical fields as Mathieu beams, Caustic beams, parabolic beams (Gutiérrez-Vega et. al. 2000; Anguiano-Morales et. al. 2007; Bandres 2004), and others generalized beams, the Bessel beams (Durnin et. al 1987; Bouchal et. al 1988) are undoubtedly the best known. During the last decade, Bessel beams have been studied extensively by many researchers due to their nondiffracting and self-healing features. Has also been shown that Laguerre-Gaussian beams and partially coherent beams also can self-regenerate (Wang et. al 2016; Vyas et. al 2011). 
In recent years, considerable attention has been paid to the Airy beams due to interesting physical properties and broad application prospects such as in particle trapping (Baumgartl et. al 2008; Rafael 2021) plasma guidance (Polynkin et. al 2009), vacuum electron acceleration (Li et. al 2010), and routing surface plasmon polaritons (Salandrino et. al 2010).

An ideal 2D Airy beam consists of two "wings", with a right angle between the two "wings" of the Airy, Airy beams are a kind of non-diffracting beams and possesses the self-healing property (Qian et. al 2014); Xingchun et. al 2018), although there is self-healing ability; recently, several authors have reviewed the propagation properties of Airy beams.

In (Yi et. al 2012) was shown that a partially blocked Airy beam cannot maintain their shapes during propagation, when the initial angle between two "wings" of the 2D Airy is non-orthogonal, then the self-healing property is absent. Chu et al. showed that the speed of self-healing process is affected by the size of the obstacle, since a large opaque obstacle causes slow reformation of the Airy beam (Xiuxiang et. al 2012). The propagation of Airy beam in turbulence was examined in (Yingjun et. al 2021; Huaili et. al 2022), results showed that the propagation through a disordered medium tend to affect the intensity distribution and the effect of self-healing ability does not succeed satisfactorily.

Nowadays, there are many types of Airy beams that are experimentally generated, such as Airy beam arrays (Yalong et. al 2010; Chen et. al 2014; Deng et. al 2013), linear, rectangular, and circular Airy beam (Wenguo et. al 2015; Zhong et. al 2016), and that deserve to be studied due to their wide applications to technical areas and their fascinating feature of transverse acceleration upon propagation (Siviloglou et. al 2007). 
On the other hand, the Airy vortex beams (Hui et. al 2018; Rafael et. al 2020) and the Airy Gaussian vortex beams (Chen et. al 2015), are another variant of Airy beams. In recent years, the study of the propagation of an Airy beam with an optical vortex is a new direction in optics.

This kind of Airy vortex beam, shows a autofocusing property, the Airy autofocusing beam via a vortex structure plays an important role, since they have a rotation during propagation caused by the topological charge.

In this paper, we investigate the propagation of Airy vortex beam generated by a combination of individual standard 2D Airy beams. We show numerically that the superposition of these beams with a non-orthogonal angle between two "wings" of the superimposed 2D Airy beam produces very special and interesting structured light beams and the self-healing property is affected.

\section{Analytical expression for an Airy beam}

The amplitude of a two-dimensional Airy beam at initial plane, involves the multiplication of two individual one-dimensional Airy functions in terms of the $\mathrm{x}$ and $\mathrm{y}$ directions and is expressed as:

$$
E(x, y, z=0)=A_{i}\left(\frac{x}{x_{0}}\right) \exp \left(a_{0} \frac{x}{x_{0}}\right) \times A_{i}\left(\frac{y}{y_{0}}\right) \exp \left(a_{0} \frac{y}{y_{0}}\right)
$$

Here, $\operatorname{Ai}(\cdot)$ denotes the Airy function, $a_{0}$ is the decay coefficient is a positive quantity, to ensure the beam carrying finite energy and thus ensure the physical realization of such beams. In which (Vallée et. al 2010): 


$$
A_{i}(X)=\frac{1}{2 \pi} \int_{-\infty}^{\infty} \exp \left(\frac{i u^{3}}{3}+i X u\right) d u
$$

To investigate the interaction of several ABs, we construct the incident light field as follow:

$$
\begin{aligned}
& E_{1}(x, y, 0)=\left[A_{i}\left(\frac{x}{x_{0}}\right) A_{i}\left(\frac{y}{y_{0}}\right) \exp \left(a_{0} \frac{x}{x_{0}}+a_{0} \frac{y}{y_{0}}\right)\right] \exp ( \pm i n \varphi) \\
& E_{2}(x, y, 0)=\left[A_{i}\left(-\frac{x}{x_{0}}\right) A_{i}\left(\frac{y}{y_{0}}\right) \exp \left(a_{0} \frac{x}{x_{0}}+a_{0} \frac{y}{y_{0}}\right)\right] \exp ( \pm i n \varphi) \\
& E_{3}(x, y, 0)=\left[A_{i}\left(\frac{x}{x_{0}}\right) A_{i}\left(-\frac{y}{y_{0}}\right) \exp \left(a_{0} \frac{x}{x_{0}}+a_{0} \frac{y}{y_{0}}\right)\right] \exp ( \pm i n \varphi) \\
& E_{4}(x, y, 0)=\left[A_{i}\left(-\frac{x}{x_{0}}\right) A_{i}\left(-\frac{y}{y_{0}}\right) \exp \left(a_{0} \frac{x}{x_{0}}+a_{0} \frac{y}{y_{0}}\right)\right] \exp ( \pm i n \varphi)
\end{aligned}
$$

Here, $\varphi$ is the phase angle, and $n$ is an integer-valued of topological charges. The intensity distribution of a four-Airy autofocusing beam at different propagation positions can be defined as a sum of four two-dimensional Airy beams:

$$
E(x, y, z)=\sum_{m} E_{m}(x, y, z)
$$

\section{Superpositions of symmetrical Airy beams}

Figure 1 shows a series of intensity distributions of conventional Airy beams travel at different propagation positions in the free space. All of these Airy beams have $90^{\circ}$ angles between their two "wings". At the input, the set of independent 2D Airy beams are separated, which accelerate in the 
mutually opposite directions after a certain propagation distance. During propagation, they converge gradually on the center with the same parameters until meet together at a certain propagation position (see Figs. 1(B), (F), (J)), which results in a sharply focusing point and a large intensity enhancement.

During a long propagation distance, we can see that it maintains its intensity pattern almost unchanged, as heart beam for three Airy beams (see Figs. 1(B)) or as a square light for four Airy beams (see Fig. $1(\mathrm{~J})$ ).

Figure 1 (D) illustrate that beyond the focusing point, they still propagate individually moving away from each other, the path of the individual Airy beams always moves along the fixed $45^{\circ}$ direction on the $\mathrm{x}-\mathrm{y}$ plane. After a longer propagation from the focal point, an inverted profile is forms and do not affect their self-accelerating direction and the shape is not different from those of the initial plane.

Now we examine the evolution process of the transverse intensity pattern of 2D Airy array beams propagating for the case of $n=2$. The topological charge has a greater impact on the shape of the main lobe, after introducing a value to the topological charge, a vortex emerges into the center of the superposed 2D Airy beam and gradually becomes dominant (see Fig. 2).

During a longer propagation this pattern always maintain a zero-central intensity and the position of the singular point does not change through the propagation, with a flow of the beams along an anticlockwise rotation direction. We believe that since the shape of the hollow at the center region is well-preserved along a long distance, it may find future applications, although it shows changes on propagation at distances very far from the focal point.

Comparing with the case of no vortex (see Figs. 1(D), 1(H) and 1(L)) beyond the focusing point, the whole profiles yield a small change in their acceleration direction (see Figs. 3(A) - 3(C)). As 
the value of the topological charge increases, the displacement becomes more and more obvious. Then, the topological charge has an effect on the interaction between Airy beams, non-diffraction, and self-healing property is not affected since they are indeed composed of 2D Airy beams, only the direction of acceleration is slightly affected.

From Fig. 4 we can detect the interaction of the superposed four 2D Airy beams, precisely before converging at the central region. Figure 4 (B), shows an intensity distribution when $n=0$, the set of Airy beams always accelerates along the direction, that initially assigned. While the Fig. 4(C) shows the case when $n=2$, as result of the orbital angular momentum, clearly can be seen that the 2D Airy beams exhibits a small lateral deflection with respect to the initial direction that they had in the input plane. Then, its self-acceleration direction has a small shift along the $\mathrm{x}$ and $\mathrm{y}$ directions. We now investigate the propagation dynamics of 2D Airy beams with non-orthogonal angles between their two "wings". In (Liang et. al 2014), was shown that when these beams present oblique angles and not orthogonal ones, they lose their non-diffraction property during propagation.

Figure 5 illustrate that beyond the focusing point, they still propagate moving away from each other. After a longer propagation from the focal point, the shape is different from those of the initial plane. A second case (see Fig. 6) is when two 2D Airy beams interfere vertically with initial angle of $75^{\circ}$ between the two "wings", and other pair of beams interfere horizontally with initial angle of $90^{\circ}$ between the two "wings". Initially, all these have the same transverse separation with respect to the center point of the $x-y$ plane. This family of optical fields, after converging in the central region, continue accelerating. 
Moreover, despite of having a pair of 2D Airy beams with two "wings" forming a $90^{\circ}$ angle, it is not easy to distinguish their inverted patterns as well as in the case when all 2D Airy beams colliding with a $90^{\circ}$ angle.

We observe that, at a smaller angle between the "wings" it accelerates faster than at $90^{\circ}$. This can be seen in Fig. 6 (D), the vertical main lobes can be seen at opposite ends, while the main lobes corresponding to the horizontal beam have traveled a shorter distance. Both accelerations can be controlled by varying the initial wing angle. On the other hand, the main lobes of the vertical Airy beams gradually tend to become weaker than the horizontal Airy beams during propagation.

\section{Conclusion}

In summary, we have numerically demonstrated the generation of hollow Airy beams through the superposition of several 2D Airy beams affected by the value of the topological charge, the new distribution exhibits a transverse rotation and is non-diverging upon propagation. Our results may be useful for controlling dynamical Airy beams for various applications. Our results show that the self-acceleration of the main lobes increases when the initial wing angle turning small.

\section{Bibliography}

Anguiano-Morales M., Martinez A., Iturbe-Castillo M. D., Chavez-Cerda S., and Alcala-Ochoa N., "Self-healing properties of a caustic optical beam," Appl. Opt. 46(34), 8284-8290 (2007).

Bandres, M.; Gutiérrez-Vega, J.; Chávez-Cerda, S. Parabolic nondiffracting optical wave fields. Opt. Lett., 29, 44-46 (2004).

Baumgartl J., Mazilu M., Dholakia K., optically mediated particle clearing using Airy wavepackets, Nat. Photonics 2, 675-678 (2008). 
Bouchal Z., Wagner J., and Chlup M., Self-reconstruction of a distorted nondiffracting beam, Opt. Commun. 151, 207 (1998).

Chen Bo, Chen Chidao, Peng Xi, Peng Yulian, Zhou Meiling, and Deng Dongmei, Propagation of sharply autofocused ring Airy Gaussian vortex beams, Opt. Exp. 23, 19288-19298 (2015).

Chen Chunyi, Yang Huamin, Kavehrad Mohsen, Zhou Zhou, Propagation of radial Airy array beams through atmospheric turbulence, Optics and Lasers in Engineering 52, 106-114 (2014).

Deng Hongchang and Yuan Libo, Generation of Airy-like wave with one-dimensional waveguide array, Opt. Lett. 38, 1645-1647 (2013).

Durnin, J.; Miceli, J.J., Jr.; Eberly, J.H. Diffraction-free beams. Phys. Rev. Lett., 58, 1499-1501. (1987).

Gutiérrez-Vega, J.; Iturbe-Castillo, M.; Chávez-Cerda, S. Alternative formulation for invariant optical fields: Mathieu beams. Opt.Lett. 25, 1493-1495 (2000).

Huaili Zhang, Haijing Deng, Xin Wang, Xiuxiang Chu, Self-healing ability of radially Airy beam, Optik - International Journal for Light and Electron Optics 251, 168478 (2022).

Hui Li, Haigang Liu, and Xianfeng Chen, Nonlinear generation of Airy vortex beam, Opt. Exp. 26, 21204-21209 (2018).

Li J., Zang W., Tian J., Vacuum laser-driven acceleration by Airy beams, Opt. Exp. 18, 7300-7306 (2010).

Liang Yi, Hu Yi, Ye Zhuoyi, Song Daohong, Lou Cibo, Zhang Xinzheng, Xu Jingjun, Morandotti Roberto, and Chen Zhigang, Dynamical deformed Airy beams with arbitrary angles between two wings, J. Opt. Soc. Am. A 31 1468-1472 (2014).

Polynkin P, Kolesik M, Moloney JV, Siviloglou GA, Christodoulides DN., Curved plasma channel generation using ultraintense Airy beams, Science 324, 229-232 (2009). 
Salandrino A., Christodoulides D.N., Airy plasmon: a nondiffracting surface wave, Opt. Lett. 35, 2082-2084 (2010).

Siviloglou G.A., Broky J., Dogariu A., Christodoulides D.N., Observation of accelerating airy beams, Phys. Rev. Lett. 99, 213901 (2007).

Qian Yixian, Wyrowski Frank, Evolution of self-healing characteristic on optical Airy beam, Optik 125, 3876-3879 (2014).

Rafael A.B. Suarez, Antonio A.R. Neves, Marcos R.R. Gesualdi, Optical trapping with nondiffracting Airy beams array using a holographic optical tweezers, Optics \& Laser Technology 135, 106678 (2021).

Rafael A.B. Suarez, Antonio A.R. Neves, Marcos R.R. Gesualdi, Generation and characterization of an array of Airy-vortex beams, Opt. Commun. 458, 124846 (2020).

Vallée Olivier, Soares Manuel, Airy Functions And Applications To Physics, Imperial College Press, London (2010).

Vyas S., Niwa M., Kozawa Y., and Sato S., "Diffractive properties of obstructed vector LaguerreGaussian beam under tight focusing condition,” J. J. Opt. Soc. Am. A 28(7), 1387-1394 (2011).

Wang F., Chen Y., Liu X. L., Cai Y. J., and Ponmarenko S. A., "Self-reconstruction of partially coherent light beams scattered by opaque obstacles," Opt. Exp. 24, 23735-23746 (2016).

Wenguo Zhu and Weilong She, Tightly focusing vector circular airy beam through a hard aperture, Opt. Commun. 334, 303-307 (2015).

Xingchun Chu, Shanghong Zhao, Yingwu Fang, Maximum nondiffracting propagation distance of aperture-truncated Airy Beams, Opt. Commun. 414, 5-9 (2018).

Xiuxiang Chu, Guoquan Zhou, and Ruipin Chen, "Analytical study of the self-healing property of Airy beams," Phys. Rev. A 85, 013815 (2012). 
Yalong Gu and Greg Gbur, Scintillation of Airy beam arrays in atmospheric turbulence, Opt. Lett. 35, 3456-3458 (2010).

Yi Hu, Georgios A. Siviloglou, Peng Zhang, Nikolaos K. Efremidis, Demetrios N. Christodoulides, and Zhigang Chen, Self-accelerating Airy Beams: Generation, Control, and Applications. In: Chen Z., Morandotti R. (eds) Nonlinear Photonics and Novel Optical Phenomena. Springer Series in Optical Sciences, vol 170. Springer, New York, NY 2012.

Yingjun He, Ying Li, Xingyuan Chen, Liting Niu, Weiling Zhu, Self-healing behavior of Airy beam scattered from a turbulent boundary layer, Optik - International Journal for Light and Electron Optics 237, 166692 (2021).

Zhong Hua, Zhang Yiqi, Milivoj R. Belić, Changbiao Li, Wen Feng, Zhang Zhaoyang, and Zhang Yanpeng, Controllable circular Airy beams via dynamic linear potential, Opt. Exp 24, 74957506. (2016).

\section{CAPTION FUGURE}

Figure 1 Propagation dynamics of conventional 2D Airy beams, (A), (E) and (I), at input plane $z=0,(\mathrm{~B}),(\mathrm{F})$ and (J) at $\mathrm{z}=8 \mathrm{~cm},(\mathrm{C}),(\mathrm{G})$ and $(\mathrm{K})$ at $\mathrm{z}=15 \mathrm{~cm},(\mathrm{D}),(\mathrm{H})$ and $(\mathrm{L})$ at $\mathrm{z}=29 \mathrm{~cm}$,

Figure 2. Snapshots of the transverse intensity patterns at different propagation distances of two 2D vortex Airy beams, three $2 \mathrm{D}$ vortex Airy beams and four $2 \mathrm{D}$ vortex Airy beams carrying $\mathrm{n}=2$ with initial wing angle $90^{\circ},(\mathrm{A})$ at $\mathrm{z}=9 \mathrm{~cm}$, (B) $\mathrm{z}=15 \mathrm{~cm},(\mathrm{C}) \mathrm{z}=9 \mathrm{~cm},(\mathrm{D}) \mathrm{z}=15 \mathrm{~cm}(\mathrm{E}) \mathrm{z}=9 \mathrm{~cm}$, and (F) $\mathrm{z}=15 \mathrm{~cm}$ respectively.

Figure 3. Propagation of 2D Airy beams with a vortex of a unit topological charge $(\mathrm{n}=2)$ imposed at $\mathrm{z}=29 \mathrm{~cm}, \mathrm{~A})$ two Airy beams, B) three Airy beams, and C) four Airy beams.

Figure 4. Propagation of four 2D Airy beams at A) $\mathrm{z}=0 \mathrm{~cm}$ (input plane), B) $\mathrm{z}=6 \mathrm{~cm}$, and C) $\mathrm{z}=6 \mathrm{~cm}$ but with a vortex of a unit topological charge $n=2$. 
Figure 5. Snapshots of the transverse intensity patterns at different propagation distances for two 2D vortex Airy beams with initial angle of $75^{\circ}$ between the two "wings". (A) at $\mathrm{z}=0 \mathrm{~cm}$, (B) $\mathrm{z}=8 \mathrm{~cm}$, (C) $\mathrm{z}=16 \mathrm{~cm}$, and (D) $\mathrm{z}=24$ $\mathrm{cm}$ respectively.

Figure 6. Transverse intensity patterns at different propagation distances for four 2D vortex Airy beams with different initial angles between the two "wings". (A) at $\mathrm{z}=0 \mathrm{~cm},(\mathrm{~B}) \mathrm{z}=8 \mathrm{~cm},(\mathrm{C}) \mathrm{z}=15 \mathrm{~cm}$, and (D) $\mathrm{z}=24 \mathrm{~cm}$ respectively. 
Figures

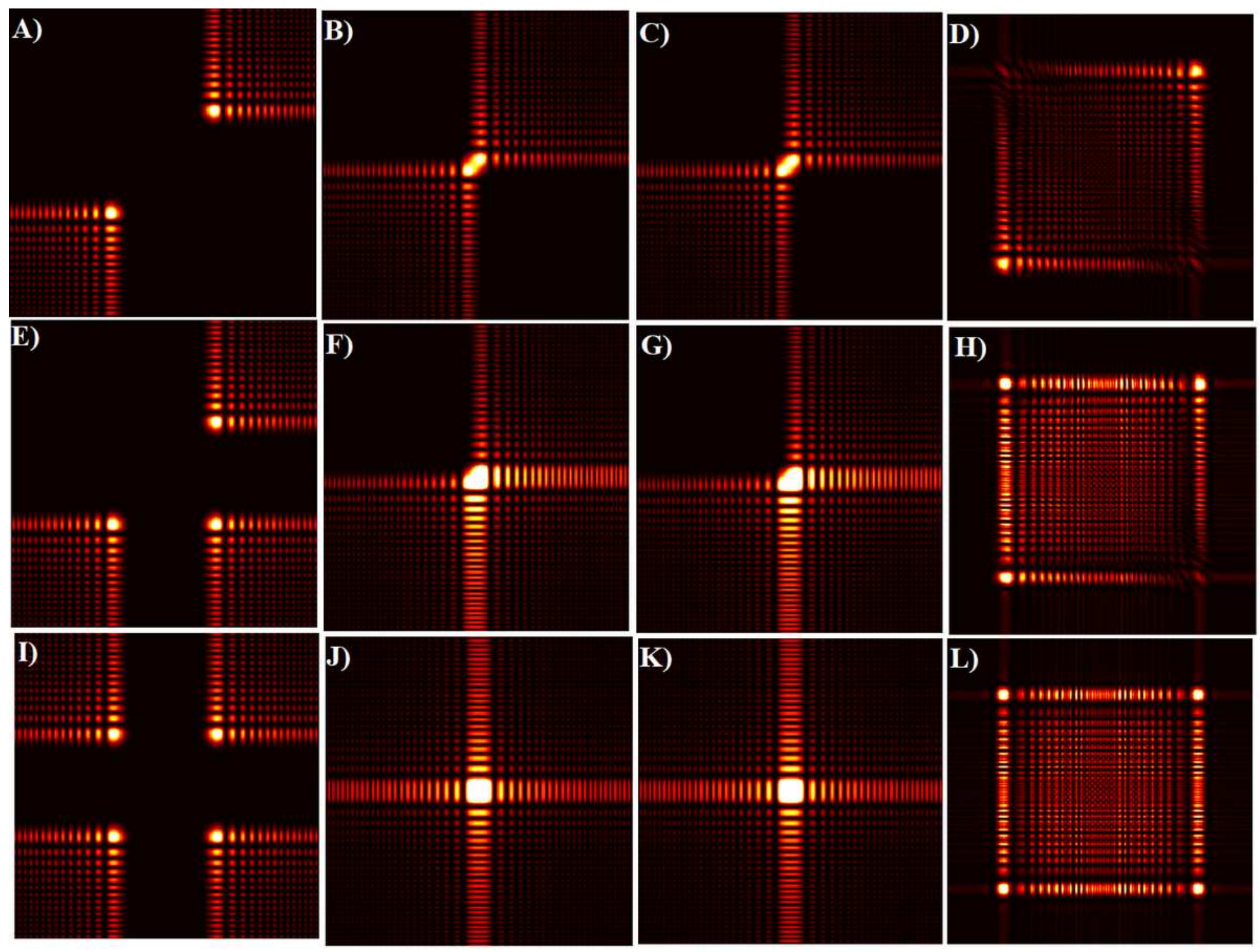

Figure 1

Propagation dynamics of conventional 2D Airy beams, (A), (E) and (I), at input plane $z=0,(B),(F)$ and $(J)$ at $z=8 \mathrm{~cm},(C),(G)$ and $(K)$ at $z=15 \mathrm{~cm},(D),(H)$ and $(L)$ at $z=29 \mathrm{~cm}$, 


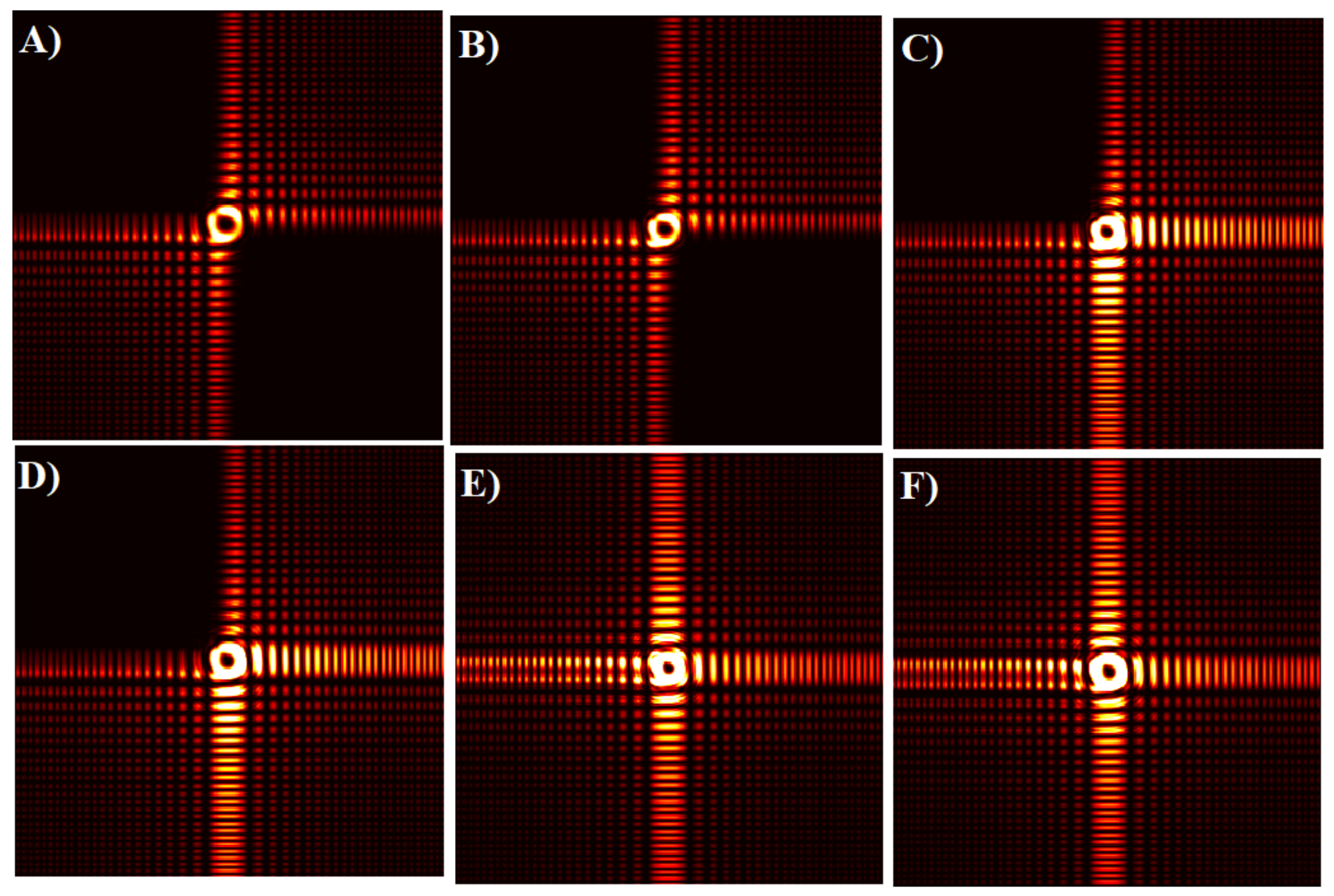

Figure 2

Snapshots of the transverse intensity patterns at different propagation distances of two 2D vortex Airy beams, three $2 \mathrm{D}$ vortex Airy beams and four $2 \mathrm{D}$ vortex Airy beams carrying $\mathrm{n}=2$ with initial wing angle $90^{\circ}$, (A) at $z=9 \mathrm{~cm},(B) z=15 \mathrm{~cm},(C) z=9 \mathrm{~cm},(D) z=15 \mathrm{~cm}(E) z=9 \mathrm{~cm}$, and (F) $z=15 \mathrm{~cm}$ respectively.

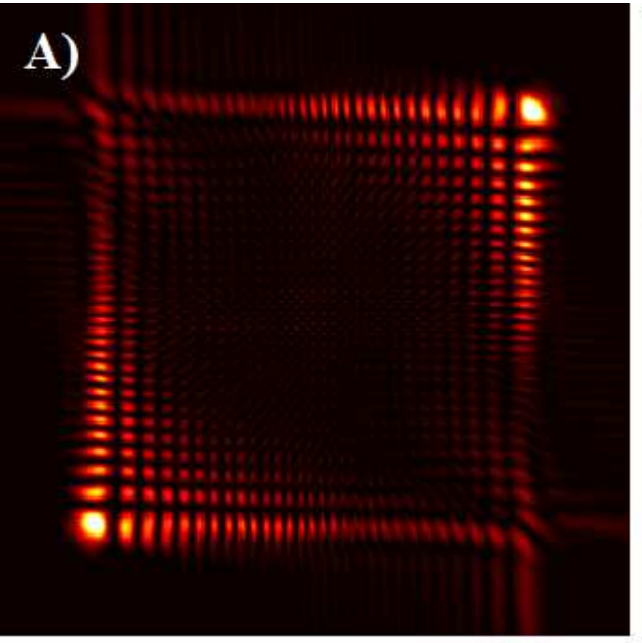

\section{B)}

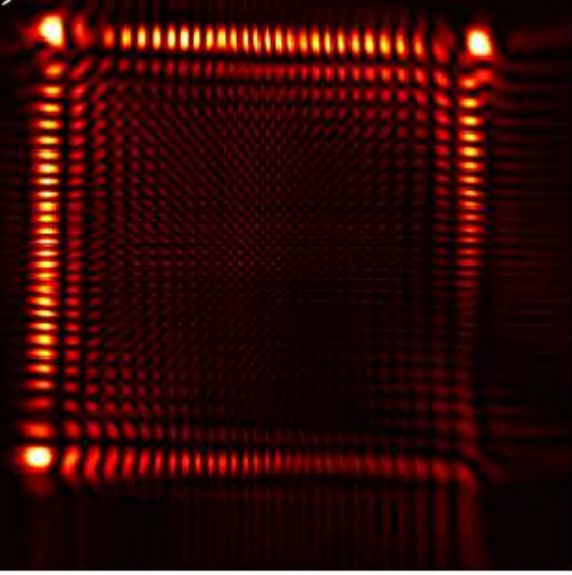

C)

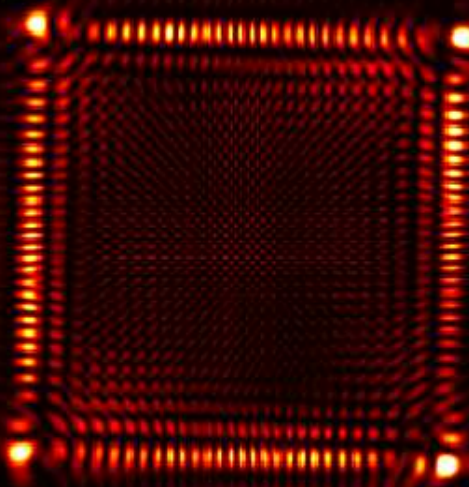

Figure 3 
Propagation of 2D Airy beams with a vortex of a unit topological charge $(n=2)$ imposed at $z=29 \mathrm{~cm}, A)$ two Airy beams, B) three Airy beams, and C) four Airy beams.
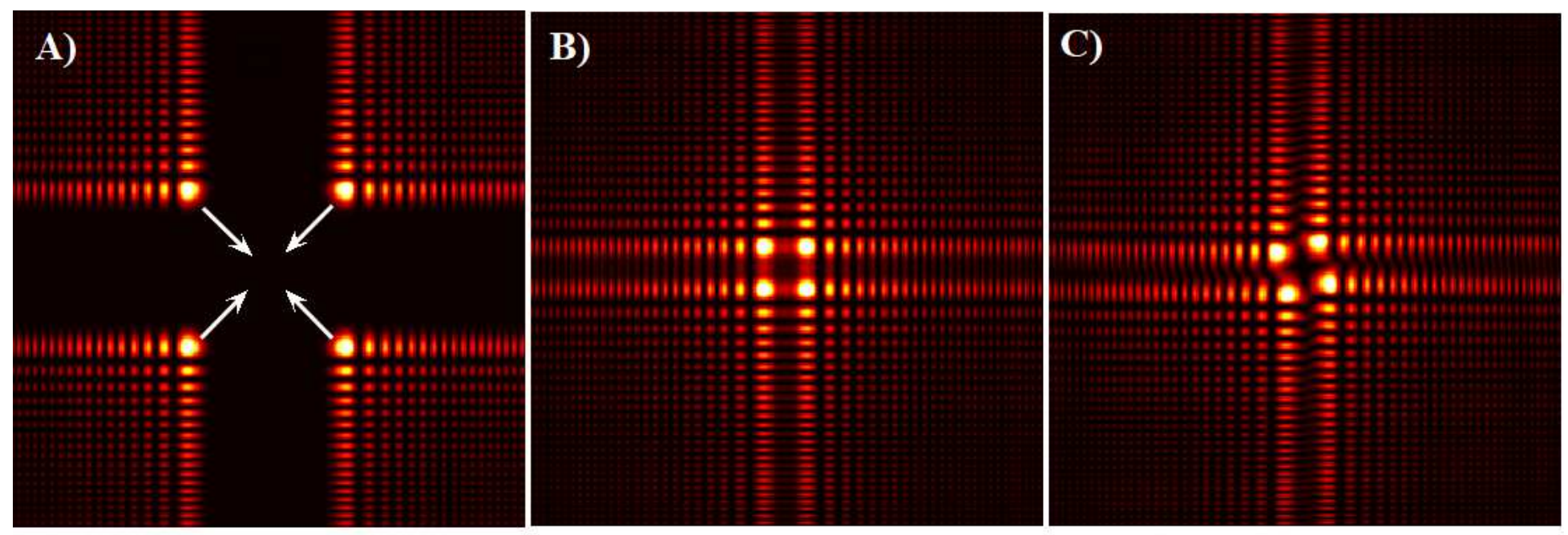

\section{Figure 4}

Propagation of four 2D Airy beams at A) $z=0 \mathrm{~cm}$ (input plane), B) $z=6 \mathrm{~cm}$, and C) $z=6 \mathrm{~cm}$ but with a vortex of a unit topological charge $n=2$. 


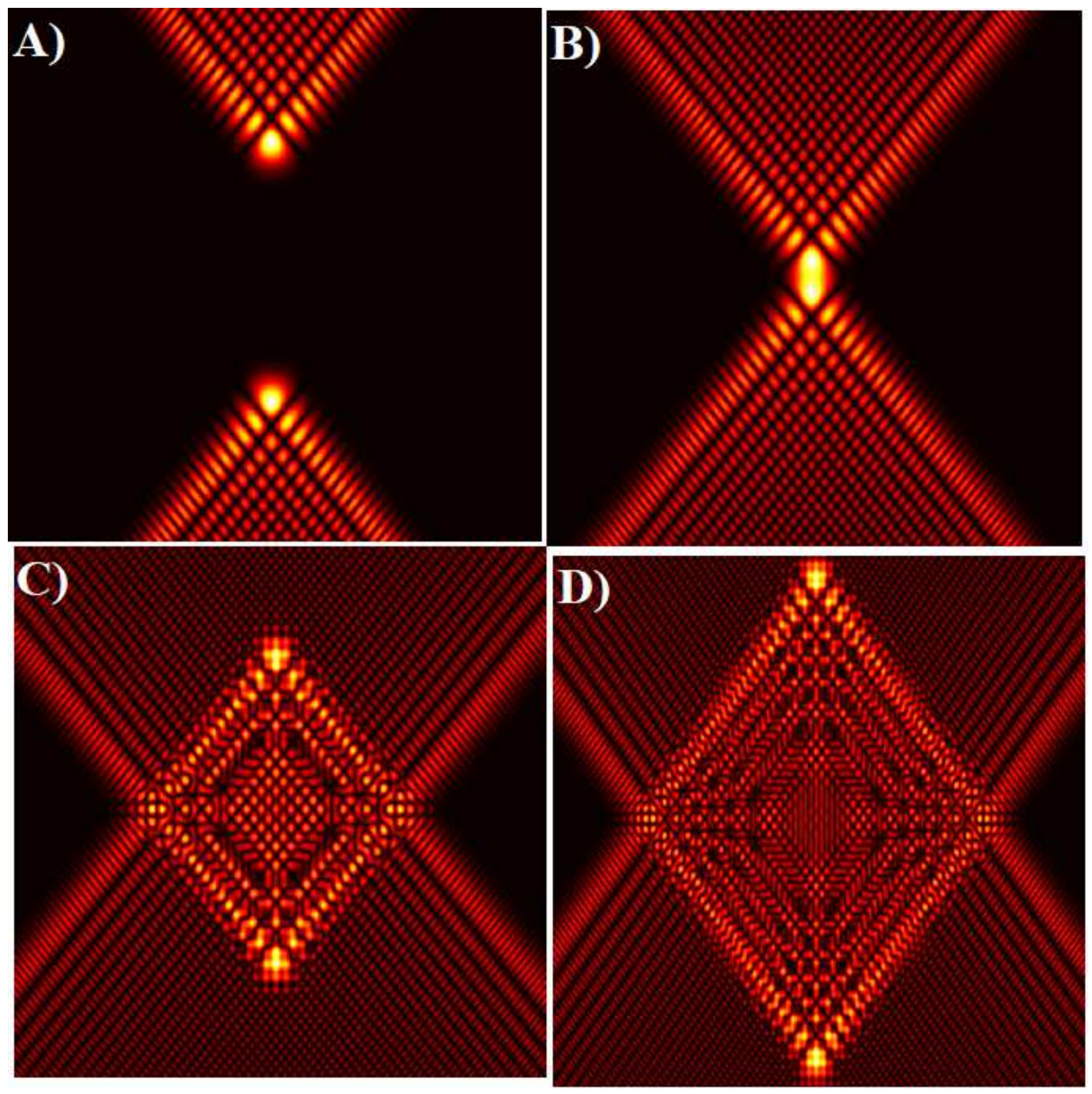

Figure 5

Snapshots of the transverse intensity patterns at different propagation distances for two 2D vortex Airy beams with initial angle of $75^{\circ}$ between the two "wings". (A) at $z=0 \mathrm{~cm},(B) z=8 \mathrm{~cm},(C) z=16 \mathrm{~cm}$, and (D) $z=24 \mathrm{~cm}$ respectively. 

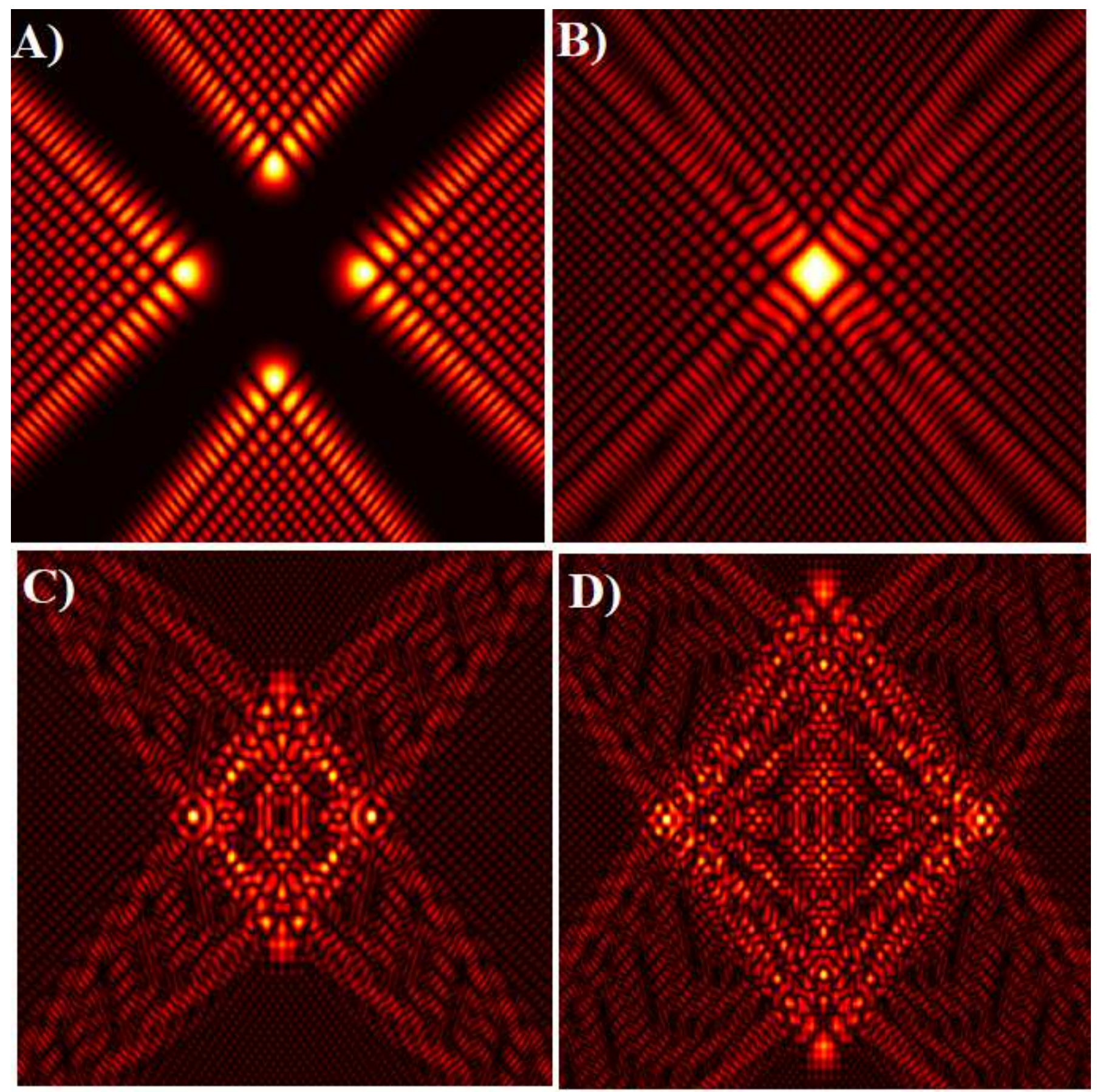

\section{Figure 6}

Transverse intensity patterns at different propagation distances for four 2D vortex Airy beams with different initial angles between the two "wings". (A) at $z=0 \mathrm{~cm},(B) z=8 \mathrm{~cm},(C) z=15 \mathrm{~cm}$, and (D) $z=24$ $\mathrm{cm}$ respectively.

\section{Supplementary Files}

This is a list of supplementary files associated with this preprint. Click to download.

- Highlights.docx 